\title{
Image Blocking Encryption Algorithm Based on Laser Chaos Synchronization
}

\author{
Shu-Ying Wang, ${ }^{1}$ Jian-Feng Zhao, ${ }^{2}$ Xian-Feng Li, ${ }^{3}$ and Li-Tao Zhang ${ }^{4}$ \\ ${ }^{1}$ Department of Nationalities, Huanghe Science and Technology College, Zhengzhou 450053, China \\ ${ }^{2}$ Department of Information Engineering, Henan Polytechnic, Zhengzhou 450046, China \\ ${ }^{3}$ College of Mathematics and Science, Lanzhou Jiaotong University, Lanzhou 730070, China \\ ${ }^{4}$ Department of Mathematics and Physics, Zhengzhou Institute of Aeronautical Industry Management, \\ Zhengzhou 450015, China
}

Correspondence should be addressed to Jian-Feng Zhao; zjfzwf@126.com

Received 27 May 2016; Accepted 10 July 2016

Academic Editor: Wen Chen

Copyright (C) 2016 Shu-Ying Wang et al. This is an open access article distributed under the Creative Commons Attribution License, which permits unrestricted use, distribution, and reproduction in any medium, provided the original work is properly cited.

In view of the digital image transmission security, based on laser chaos synchronization and Arnold cat map, a novel image encryption scheme is proposed. Based on pixel values of plain image a parameter is generated to influence the secret key. Sequences of the drive system and response system are pretreated by the same method and make image blocking encryption scheme for plain image. Finally, pixels position are scrambled by general Arnold transformation. In decryption process, the chaotic synchronization accuracy is fully considered and the relationship between the effect of synchronization and decryption is analyzed, which has characteristics of high precision, higher efficiency, simplicity, flexibility, and better controllability. The experimental results show that the encryption algorithm image has high security and good antijamming performance.

\section{Introduction}

With the rapid development of information transmission technology, electronic data interchange is an important way to communicate and exchange information. Multimedia information with secure communication problems urgently needs to be solved. Characteristics of chaotic sequence have natural advantages in cryptography. During the late 1980s, the British mathematician Matthews put forward the chaos into cryptography firstly [1]. As the traditional encryption technique DES cannot be directly used for image encryption, recently many special properties of chaotic maps are suitable to encrypt digital image and provide a new idea to select data security problem of multimedia information [2-7].

The laser chaos not only has complex phenomena of all dissipative systems, but also has excellent characteristics such as built-in self-test ability (BIST), pulse generation, closeness to the ideal model, and easiness of design compared with theory. Chaotic laser signal with inherent broad band, noise, and unpredictable characteristics greatly increased the difficulty of optoelectronic reconnaissance and is an important technology of optical information security. The laserbased digital communication was put forward by Colet and Roy in 1994 [8] and became increasingly popular in recent years [9-11].

Synchronization is a basic characteristic of complex system and the phenomenon generally exists in natural ecosystem and artificial system such as biology, engineering, and machinery $[12,13]$. Synchronization of chaotic systems and secure communication have become research hotspots of nonlinear system $[14,15]$. With simple structure the linear feedback controller is easily implemented physically and has high practical value, but chaos synchronization is rarely applied in digital image encryption $[16,17]$.

This paper is organized as follows. In Section 2, linear feedback synchronization of Lorenz-Haken is introduced. In Section 3, based on chaos synchronization, a novel image encryption algorithm is proposed. In Section 4, numerical simulation is given to illustrate the effectiveness of the proposed algorithm. Finally, the paper concludes in Section 5. 


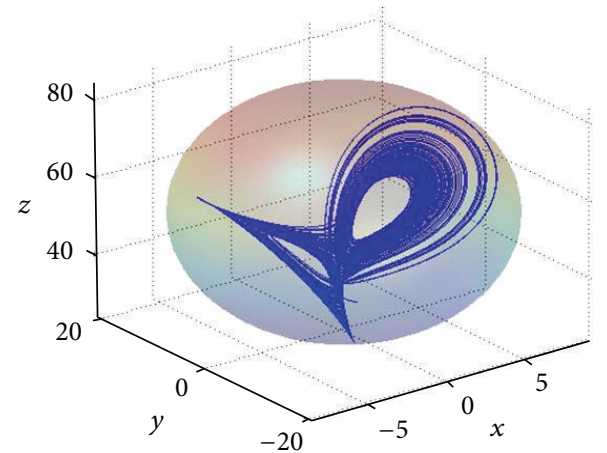

(a) Phase space
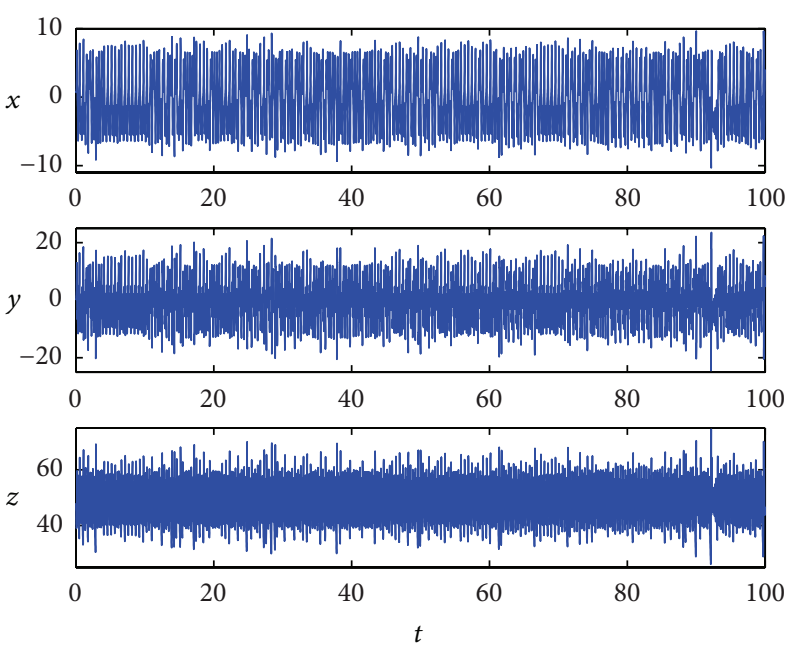

(b) Chaos sequences

FIGURE 1: Laser chaos.

\section{Linear Feedback Synchronization of Lorenz-Haken Laser Chaos}

The mathematical description of the Lorenz-Haken laser chaos [20] is

$$
\begin{aligned}
& \dot{x}=a(y-x), \\
& \dot{y}=b x-y-x z, \\
& \dot{z}=x y-c z,
\end{aligned}
$$

where $X=(x, y, z)^{T}$ is state vector of system (1) and $a, b, c$ are system parameters assigned to $(a, b, c)=(1.4253,50,0.2775)$. Initial conditions are $\left(x_{0}, y_{0}, z_{0}\right)=(1,0,50)$ and time is $t \in$ $[0,100 s]$.

In view of the boundary of chaotic attractor, there are positive numbers $M_{x}, M_{y}$, and $M_{z}$, and $|x| \leq M_{x},|y| \leq M_{y}$, and $|z| \leq M_{z}$. Figure 1 shows the phase diagram of chaotic system and state variables which meet the range $-9<x<9$, $-21<y<21$, and $21<z<81$.

System (1) is used as the drive system, and the response system is defined as follows:

$$
\begin{aligned}
& \dot{x}_{1}=a\left(y_{1}-x_{1}\right)+u_{1}, \\
& \dot{y}_{1}=b x_{1}-y_{1}-x_{1} z_{1}+u_{2}, \\
& \dot{z}_{1}=x_{1} y_{1}-c z_{1}+u_{3} .
\end{aligned}
$$

Suppose that error variables of the system are $e_{1}=x-x_{1}$, $e_{2}=y-y_{1}$, and $e_{3}=z-z_{1}$. A linear feedback controller is selected as $u_{i}=-k_{i} e_{i}, \quad(i=1,2,3)$.

Then we get the error system:

$$
\begin{aligned}
& \dot{e}_{1}=\left(k_{1}-a\right) e_{1}+a e_{2}, \\
& \dot{e}_{2}=b e_{1}+\left(k_{2}-1\right) e_{2}+x z-x_{1} z_{1}, \\
& \dot{e}_{3}=\left(k_{3}-c\right) e_{3}-x y+x_{1} y_{1} .
\end{aligned}
$$

The Lyapunov function is designed as $V=(1 / 2)\left(e_{1}^{2}+\right.$ $\left.e_{2}^{2}+e_{3}^{2}\right)$; then tracking along system (3), we obtain its time derivative:

$$
\begin{aligned}
\dot{V}= & e_{1} \dot{e}_{1}+e_{2} \dot{e}_{2}+e_{3} \dot{e}_{3} \\
= & \left(k_{1}-a\right) e_{1}^{2}+a e_{1} e_{2}+b e_{1} e_{2}+\left(k_{2}-1\right) e_{2}^{2} \\
& +\left(k_{3}+b\right) e_{3}^{2}+\left(x z-x_{1} z_{1}\right) e_{2}+\left(k_{3}-c\right) e_{3}^{2} \\
& +\left(x_{1} y_{1}-x y\right) e_{3} \\
= & \left(k_{1}-a+\frac{(a+b-z)^{2}+y^{2}}{4}\right) e_{1}^{2}+k_{2} e_{2}^{2} \\
& +\left(k_{3}-c+1\right) e_{3}^{2}-\left(e_{2}-\frac{a+b-z}{2} e_{1}\right)^{2} \\
& -\left(e_{3}-\frac{y}{2} e_{1}\right)^{2} .
\end{aligned}
$$

To obtain the asymptotic stability of error system, that is to say, $\dot{V} \leq 0$, the following conditions need to be satisfied:

$$
\begin{aligned}
k_{1}-a+\frac{(a+b-z)^{2}+y^{2}}{4} & <0, \\
k_{2} & <0, \\
k_{3}-c+1 & <0 .
\end{aligned}
$$

Sufficient conditions of the feedback gains $k_{1}, k_{2}$, and $k_{3}$ are

$$
\begin{aligned}
& k_{1}<a-\frac{(a+b)^{2}+M_{z}^{2}+M_{y}^{2}}{4}, \\
& k_{2}<0, \\
& k_{3}<c-1 .
\end{aligned}
$$




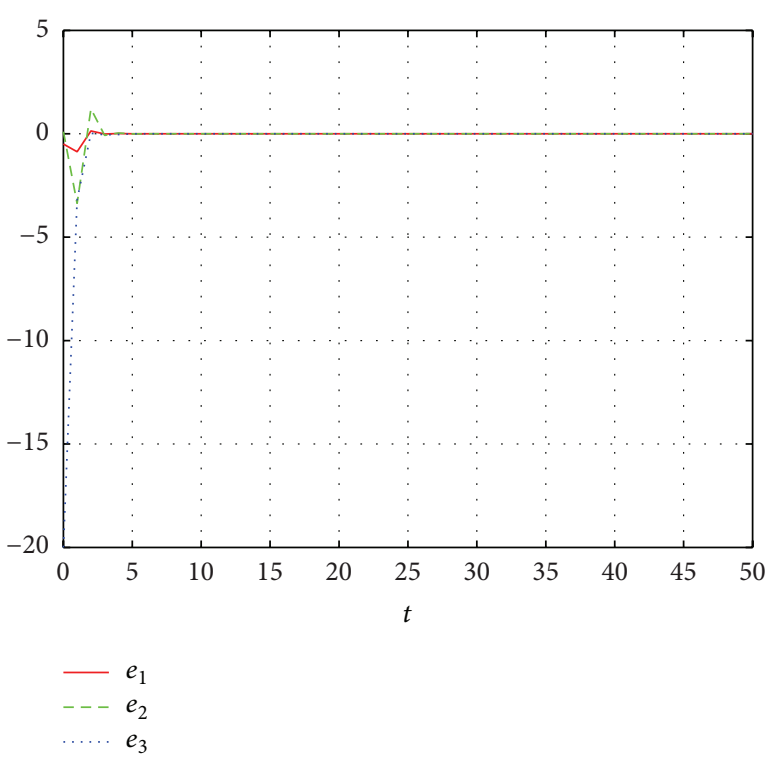

(a) Error curve $e$

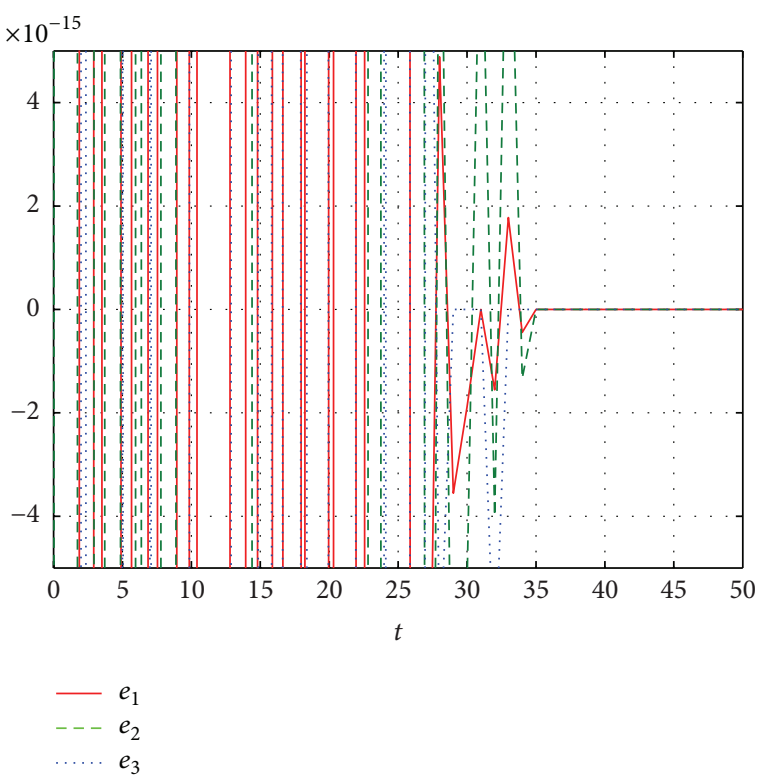

(b) Local amplification of (a)

FIGURE 2: The synchronization error curves between drive system and response system.

TABLE 1: Components of matrix $B$.

\begin{tabular}{lccc}
\hline$m=0$ & $m=1$ & $m=2$ & $m=3$ \\
\hline$B=\{B, x, y, z\}$ & $B=\{B, z, x, y\}$ & $B=\{B, y, z, x\}$ & $B=\{B, x, z, y\}$ \\
\hline
\end{tabular}

In numerical simulation experiment, the slave system is considered with the same parameters as the master system. Initial state variables of them are $(x(0), y(0), z(0))=(1,0,30)$ and $(x(0), y(0), z(0))=(1,0,30)$, respectively. Figure 2 presents the synchronization error curves between drive system and response system which approaches to zero gradually. Completely synchronization is achieved in five seconds by using macroscopic observation, but in fact there is a synchronization error. The enlargement of partial positions of error curve is shown in Figure 2(b).

\section{Description of the Algorithm}

The original image of size $L=M \times N$, where $M$ and $N$ denote numbers of row and column, respectively. By scanning line by line, the original image is rearranged into matrix $P$ :

(1) Parametric perturbation is defined by adopting for$\operatorname{mula} T=\bmod \left(\sum_{i=1}^{L} P(i), L\right) /(L-1)$. Parameters $x_{0}$, $y_{0}, z_{0}, a, b, c$, and $r$ are selected as secret key; $\{x, y, z\}$ are obtained by every chaos iteration. Then $n_{0}=$ 1000 data points are abandoned and state variables are recombined. Let $m=\bmod \left(\operatorname{abs}\left(x\left(n_{0}: n_{0}+L-1\right)+\right.\right.$ $\left.\left.y\left(n_{0}: n_{0}+L-1\right)+z\left(n_{0}: n_{0}+L-1\right)\right), 3\right)$, and extend zero matrix $B$ in Table 1 .
(2) The sequence $B$ is processed as follows:

$K(i)$

$$
=\bmod \left(\text { floor }\left((|B(i)|-\text { floor }(|B(i)|)) \times 10^{m_{2}}\right), 256\right) \text {, }
$$

where $m_{2} \in Z^{+}, 1 \leq m_{2} \leq 15$, and $i=1,2, \ldots, L$. $|x|$ is absolute of $x$, and floor $(x)$ indicates down integral function. Obviously, $K(i) \in[0,255]$ after modular arithmetic. In the paper positive integral $m_{2}=10$. Chaotic sequence $B$ shown in Figure 3(a) is treated as $K$; self-correlation of $K$ is approximate to zero presented in Figure 3(b). Figure 3(c) displays the local amplification at longitudinal amplification, and most of data are concentrated in interval $[-0.002,0.002]$. After preprocessing the randomness of chaotic sequence is improved distinctly, and sequence after treatment is more suitable in cryptography.

(3) Exclusive OR operation and modular arithmetic are imposed to the plain image by using sequence $K$. The first pixel point is encrypted individually, and its encryption exerts effect on encryption of the second pixel. The pixels are encrypted alternately as follows:

$$
\begin{aligned}
& C(2) \\
& =[P(2)+K(2)] \bmod 256 \\
& \quad \oplus[C(1)+K(L)] \bmod 256
\end{aligned}
$$

for $i=3: 2: L$ 


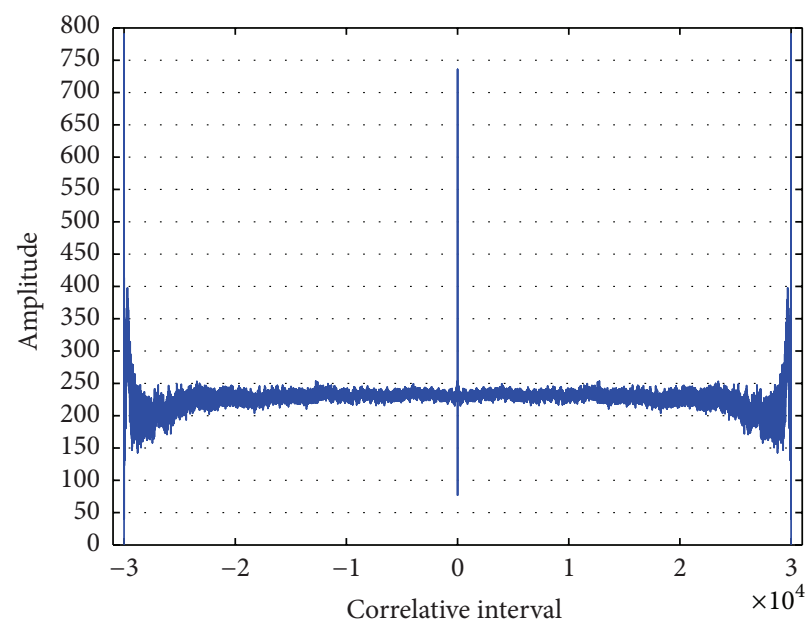

(a) Sequence $B$

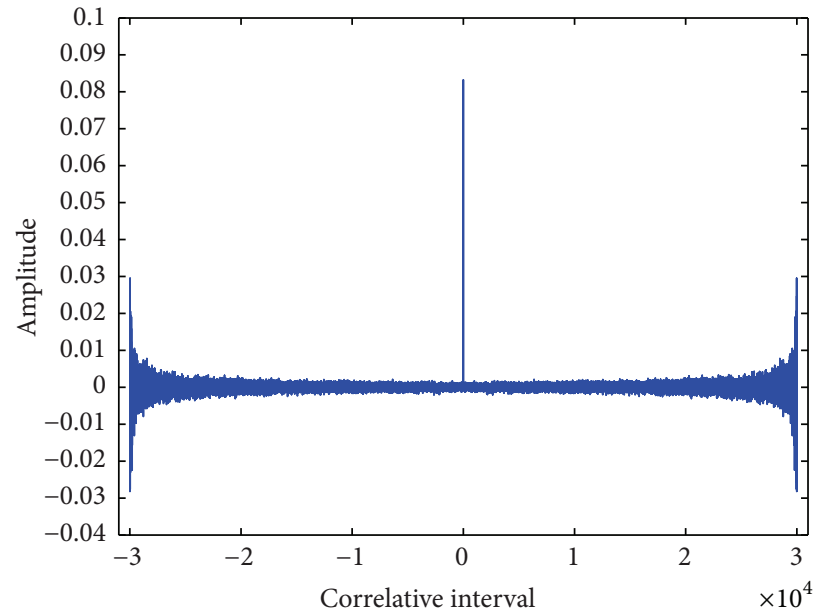

(b) Sequence $K$

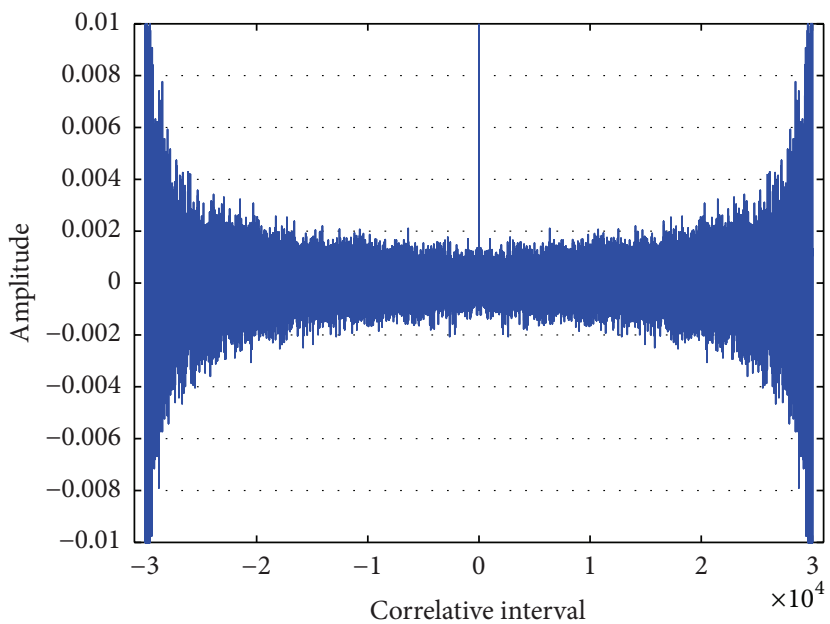

(c) Local amplification of sequence $K$

FIGURE 3: Self-correlation of chaotic sequence before and after treatment.

$C(i)$

$$
\begin{aligned}
= & {[P(i)+K(i)] \bmod 256 } \\
& \oplus[C(i-2)+K(i+L)] \bmod 256
\end{aligned}
$$

end

for $i=4: 2: L$

$$
C(i)
$$$$
=[P(i)+K(i)] \bmod 256
$$$$
\oplus[C(i-2)+K(L-i)] \bmod 256
$$

end

The encrypted pixel sequence $\{C(i)\}$ is transformed into matrix $C$ of size $M \times N$ with reshape command.

(4) In the process of scrambling, general Arnold transform is used to scramble image $C$ and get scrambled image $D$. RGB segments of color digital image are scrambled by general Arnold transform with different parameters.

For the given symmetric algorithm, decryption is the inverse operation of encryption, described as follows:

(1) The final encrypted image $D$ is inversely scrambled to get image $C$.

(2) Chaotic sequences of response system are used to perform antisubstitution decryption operation; the calculation formula is as follows:

for $i=L:-2: 3$

$P(i)$

$=\bmod (C(i) \oplus \bmod (C(i-2) \oplus K(i+L), 256), 256)$

$\oplus K(i)$ 


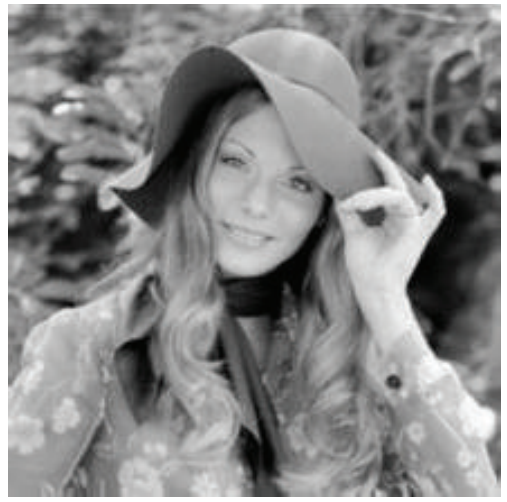

(a) Plain image

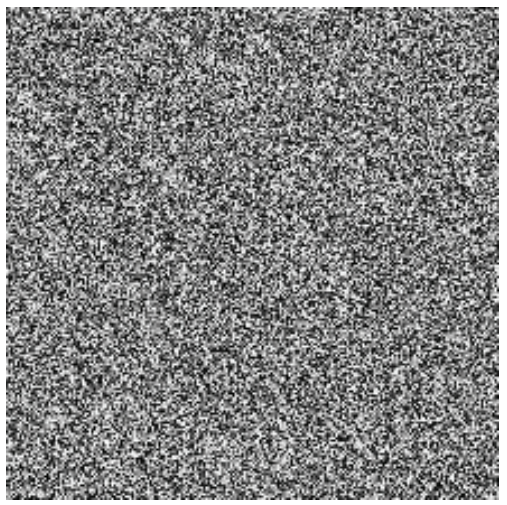

(c) Encrypted image

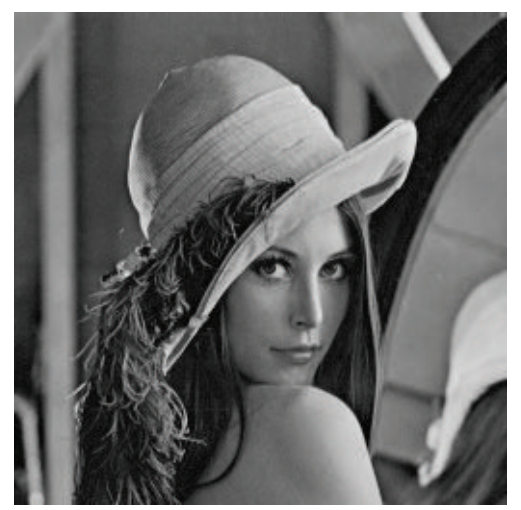

(e) Plain image

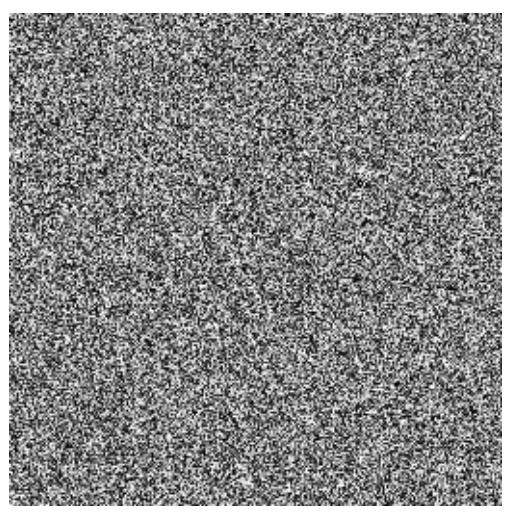

(g) Encrypted image

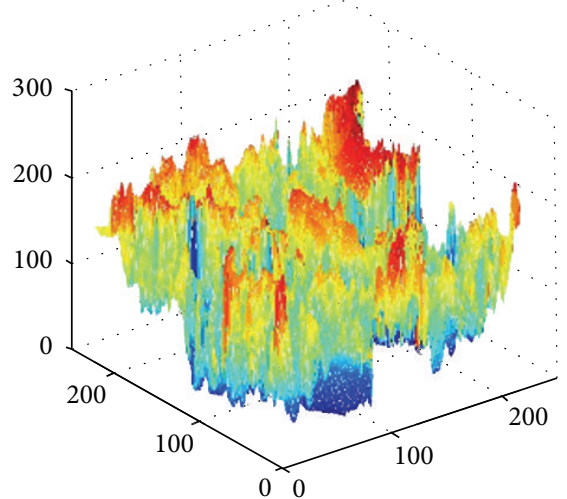

(b) Histogram of (a)

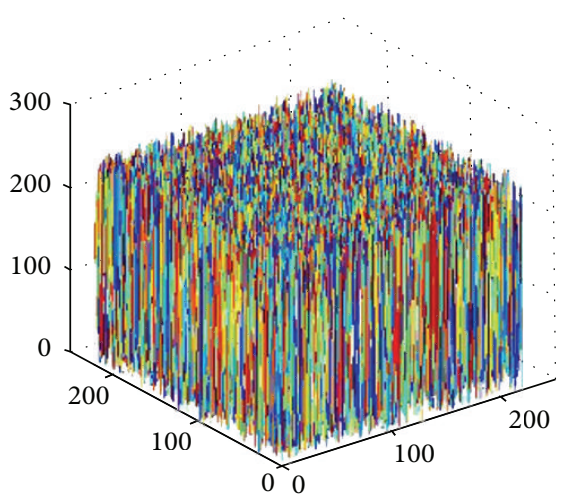

(d) Histogram of (c)

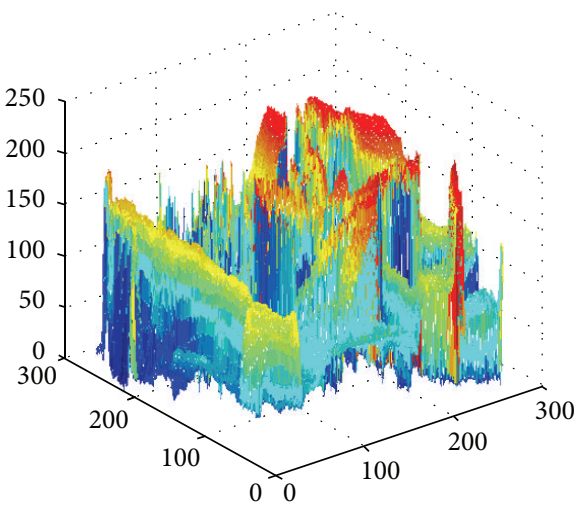

(f) Histogram of (e)

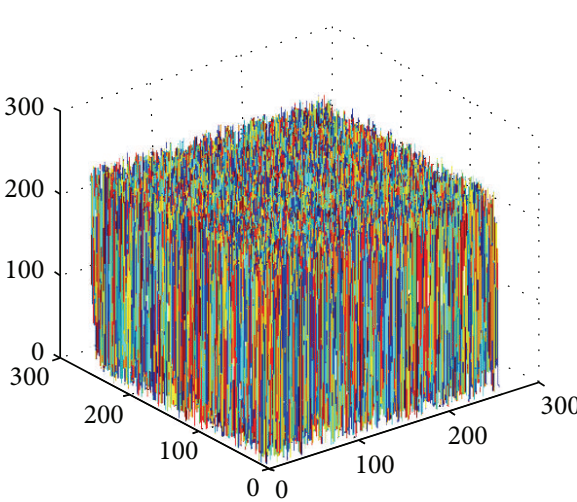

(h) Histogram of (g)

Figure 4: Continued. 


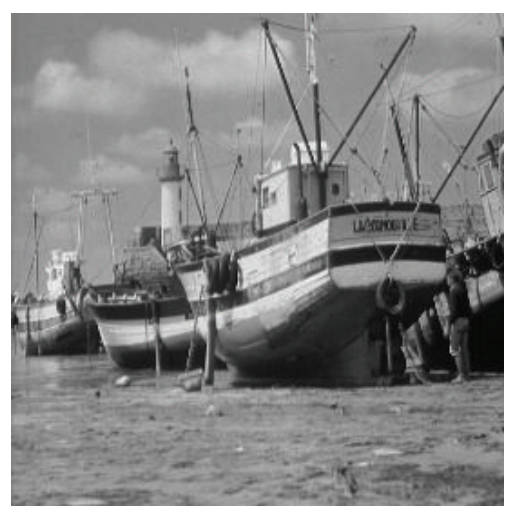

(i) Plain image

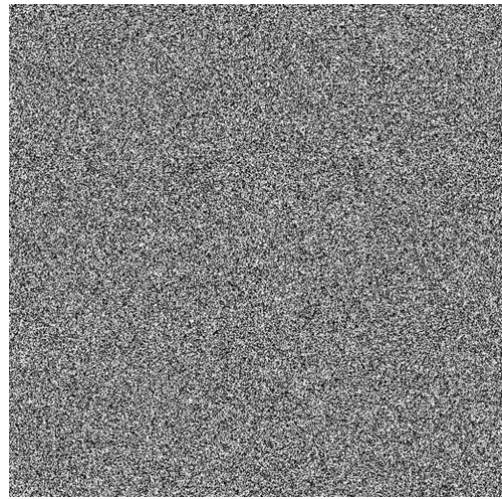

(k) Encrypted image

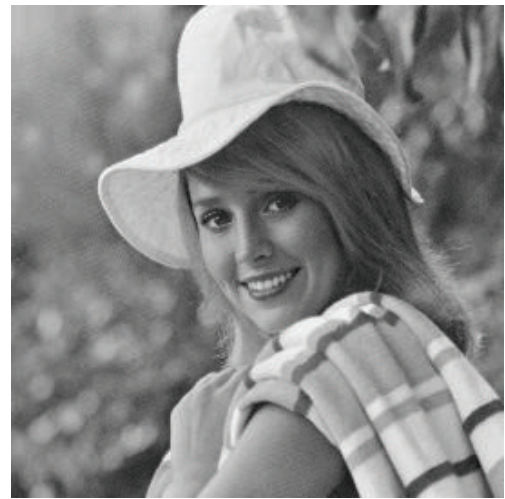

(m) Plain image

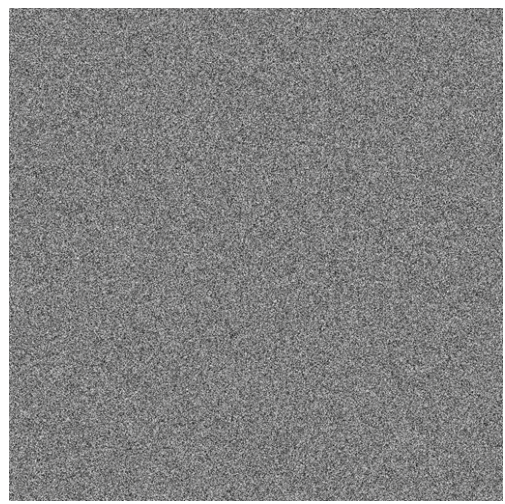

(o) Encrypted image

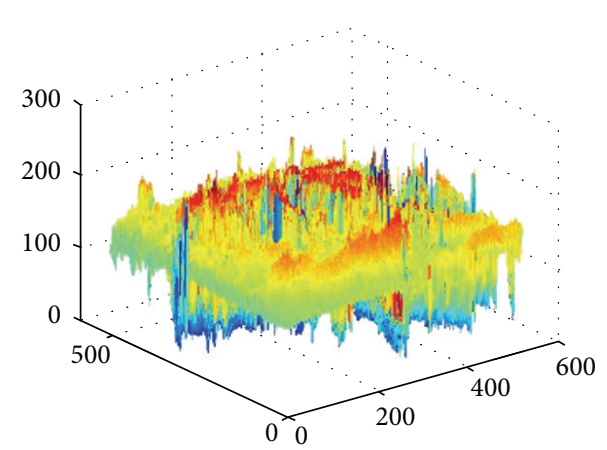

(j) Histogram of (i)

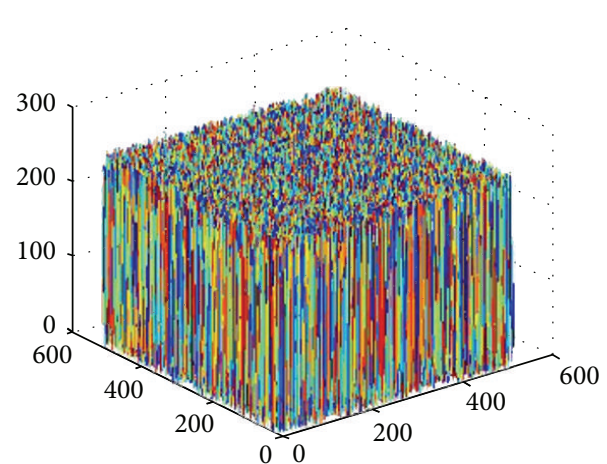

(l) Histogram of (k)

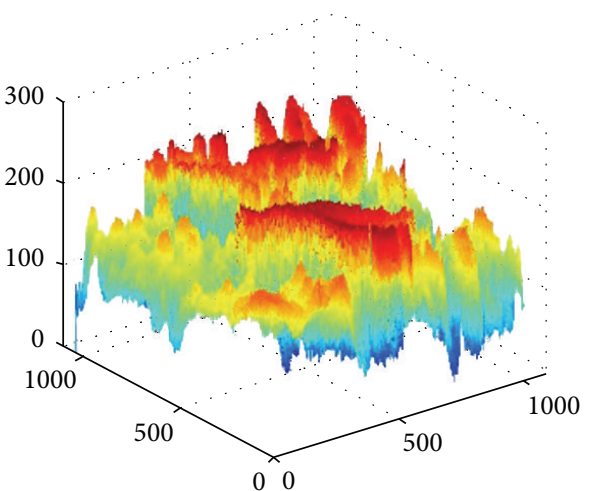

(n) Histogram of (m)

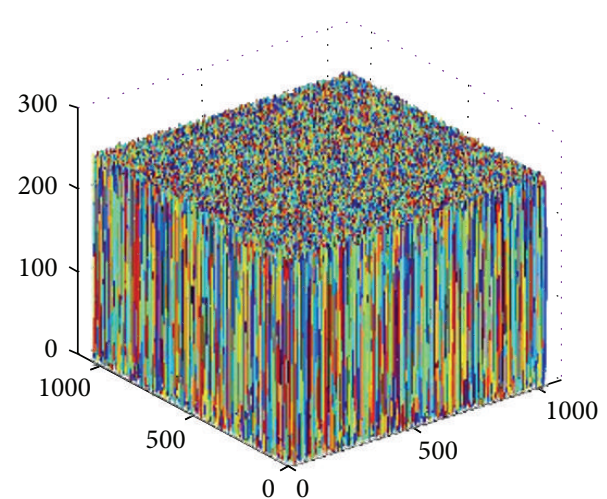

(p) Histogram of (o)

FIgure 4: Continued. 


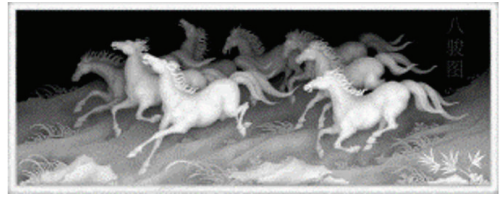

(q) Plain image

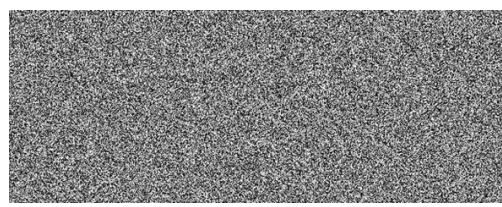

(s) Encrypted image

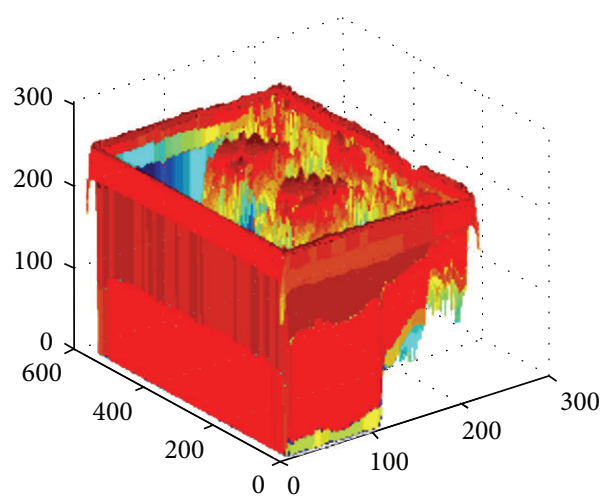

(r) Histogram of $(q)$

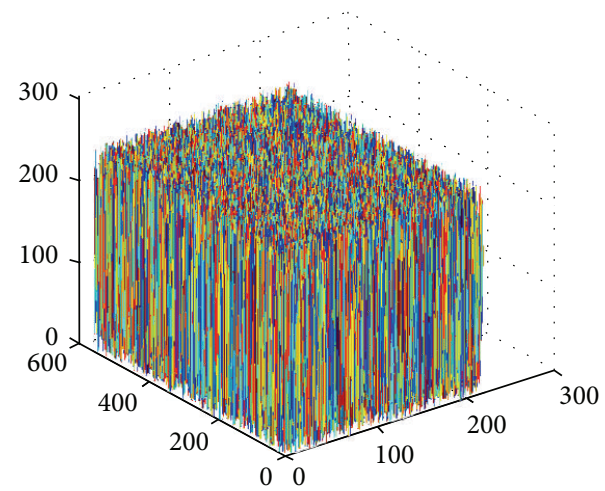

(t) Histogram of (s)

FIGURE 4: The histograms of gray images with different sizes: (a) $220 \times 220$; (e) $256 \times 256$; (i) $512 \times 512$; (m) $1024 \times 1024$; and (q) $220 \times 565$.

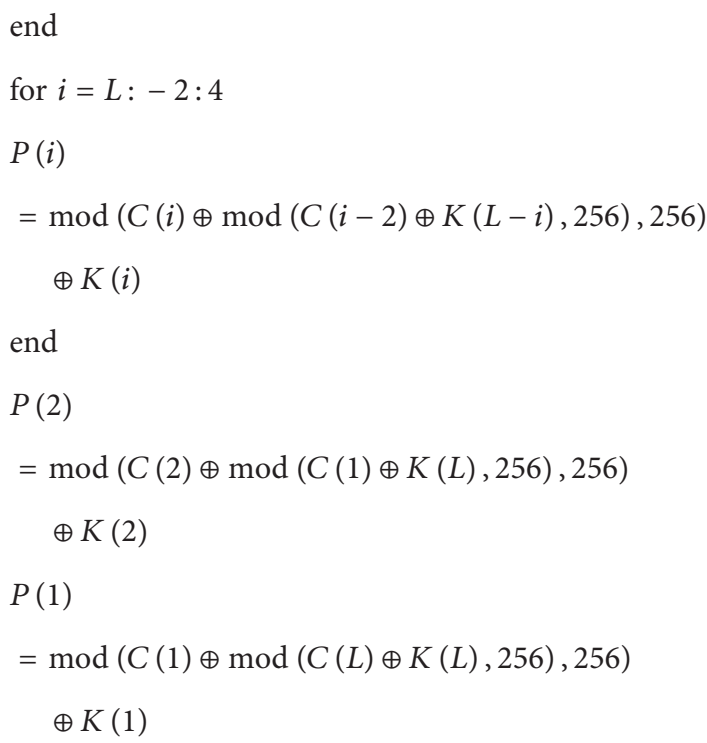

In encryption algorithm, the digital image is stored as two-dimensional array, including pixel position scrambling and pixel value substitution. For an image of size $L=M \times N$, the total time complexity is $O(L)$ analyzed from the viewpoint of the "big-O notation." Therefore, the efficiency of execution of the algorithm is ideal.

\section{Numerical Simulation Results}

To verify the effectiveness of the above algorithm, different types of images are carried out in experimentation by using MATLAB platform. Statistical histogram describes the distribution of image pixel. Under the ideal condition, histogram of encrypted image should be approximate to distribute evenly. Histograms of gray image, binary image, and color image and their encrypted images are proposed in Figures 4, 5, and 6, respectively. The eight fine horses gray image is a high resolution image with 370 dpi shown in Figure 4(q). We can see that the gray pixel values of the original image are concentrated in some values, but the histograms of different encrypted images are highly uniform.

4.1. Correlation Coefficients of Adjacent Pixels. Encryption algorithm is designed to reduce the correlation coefficients of adjacent pixels between plain image and encrypted image for resisting statistical attacks. Correlation coefficients of entire randomly selected 3000 pairs of horizontally, vertically, diagonally, and counterdiagonally adjacent pixels are determined. The correlation coefficients between two adjacent pixels of image are calculated by the following formula:

$$
R_{x y}=\frac{\operatorname{Conv}(x, y)}{\sqrt{D(x)} \sqrt{D(y)}},
$$


TABLE 2: Correlation coefficient of different plain images and cipher images.

\begin{tabular}{|c|c|c|c|c|}
\hline Plain image & Horizontal & Vertical & Diagonal & Counterdiagonal \\
\hline \multicolumn{5}{|l|}{ Gray girl $(220 \times 220)$} \\
\hline Plain image & 0.972389 & 0.973428 & 0.946752 & 0.949101 \\
\hline Encrypted image & $-2.342213 \times 10^{-4}$ & $1.178432 \times 10^{-4}$ & $4.210485 \times 10^{-5}$ & $-1.094222 \times 10^{-4}$ \\
\hline \multicolumn{5}{|l|}{ Gray Lena $(256$ × 256$)$} \\
\hline Plain image & 0.972953 & 0.970462 & 0.916925 & 0.938441 \\
\hline Encrypted image & $-4.156125 \times 10^{-4}$ & $-2.552108 \times 10^{-4}$ & $-2.788174 \times 10^{-5}$ & $-4.829102 \times 10^{-7}$ \\
\hline Encrypted image [18] & 0.000848277 & 0.00370914 & -0.000189 & \\
\hline Encrypted image [19] & -0.0025 & -0.0006 & -0.0050 & \\
\hline \multicolumn{5}{|l|}{ Gray boat $(512 \times 512)$} \\
\hline Plain image & 0.833645 & 0.941003 & 0.850811 & 0.833801 \\
\hline Encrypted image & $-1.234977 \times 10^{-4}$ & $-2.219543 \times 10^{-5}$ & $3.325240 \times 10^{-5}$ & $7.915527 \times 10^{-5}$ \\
\hline \multicolumn{5}{|l|}{ Binary image $(256 \times 256)$} \\
\hline Plain image & 0.986849 & 0.984293 & 0.965644 & 0.995392 \\
\hline Encrypted image & 0.001569 & $1.869499 \times 10^{-4}$ & $-1.574389 \times 10^{-4}$ & $-1.099277 \times 10^{-4}$ \\
\hline \multicolumn{5}{|l|}{ Gray Elaine $(1024 \times 1024)$} \\
\hline Plain image & 0.978254 & 0.966275 & 0.956931 & 0.955351 \\
\hline Encrypted image & $8.903521 \times 10^{-4}$ & $1.018005 \times 10^{-4}$ & $6.933169 \times 10^{-5}$ & $7.263636 \times 10^{-4}$ \\
\hline \multicolumn{5}{|c|}{ Eight fine horses $(220 \times 565)$} \\
\hline Plain image & 0.989809 & 0.983595 & 0.972294 & 0.972537 \\
\hline Encrypted image & $5.485211 \times 10^{-4}$ & $1.546712 \times 10^{-4}$ & $-7.799651 \times 10^{-5}$ & $-8.218992 \times 10^{-4}$ \\
\hline \multicolumn{5}{|c|}{ Color baboon $(512 \times 512 \times 3)$} \\
\hline \multicolumn{5}{|c|}{ Red } \\
\hline Plain image & 0.772788 & 0.583314 & 0.573087 & 0.509744 \\
\hline Encrypted image & $3.495943 \times 10^{-4}$ & $8.087366 \times 10^{-6}$ & $2.572029 \times 10^{-4}$ & $-4.644542 \times 10^{-5}$ \\
\hline \multicolumn{5}{|l|}{ Green } \\
\hline Plain image & 0.807396 & 0.654726 & 0.650771 & 0.597617 \\
\hline Encrypted image & $-5.508956 \times 10^{-4}$ & $-4.001587 \times 10^{-4}$ & $7.277112 \times 10^{-5}$ & $4.782903 \times 10^{-4}$ \\
\hline \multicolumn{5}{|l|}{ Blue } \\
\hline Plain image & 0.877479 & 0.790278 & 0.786803 & 0.761251 \\
\hline Encrypted image & $-7.206039 \times 10^{-5}$ & $4.348691 \times 10^{-4}$ & $-8.564954 \times 10^{-5}$ & $-4.391072 \times 10^{-4}$ \\
\hline
\end{tabular}

where

$$
\begin{aligned}
E(x) & =\frac{1}{N} \sum_{i=1}^{N} x_{i}, \\
D(x) & =\frac{1}{N} \sum_{i=1}^{N}\left[x_{i}-E(x)\right]^{2}, \\
\operatorname{Conv}(x, y) & =\frac{1}{N} \sum_{i=1}^{N}\left[x_{i}-E(x)\right]\left[y_{i}-E(y)\right],
\end{aligned}
$$

where $x$ and $y$ are pixel values of two adjacent pixels in the image.

Figure 7 and Table 2 display distribution of the randomly selected pairs of adjacent pixels in four directions of the original and encrypted image. If the correlation of the encrypted images is close to zero, then it informs good encryption quality. It is clear that the correlation coefficient of the proposed algorithm is smaller than that of other methods proposed in $[18,19]$.
4.2. Information Entropy. The concept information entropy was put forward by Shannon [21], which lays the foundation for information theory and digital communication. Information entropy is commonly used to express image texture features and measure the randomness. The entropy $H(x)$ is measured by the formula $H(x)=-\sum_{i=1}^{n} p\left(x_{i}\right) \log _{2} p\left(x_{i}\right)$, where $p\left(x_{i}\right)$ denotes the probability of symbol $x_{i}$. In theory, the maximum entropy is $H_{\max }=-(1 / 256) \log _{2}(1 / 256) \times 256=$ 8 for a gray image.

From Table 3, it is clear that our approach can encrypt a low entropy image to get higher entropy image which has random information content. The entropy of the encrypted image is actually closer to the maximum entropy than the plain image. The gray distribution of the encrypted image is more uniform, and its security is improved greatly. Obviously the encryption algorithm has better ability to resist statistical attack.

4.3. Decryption Effect. In numerical simulation, encrypted gray Lena image is decrypted by abandoning 0 points, 1000 points, 3000 points, 5000 points, and 7000 points, and 


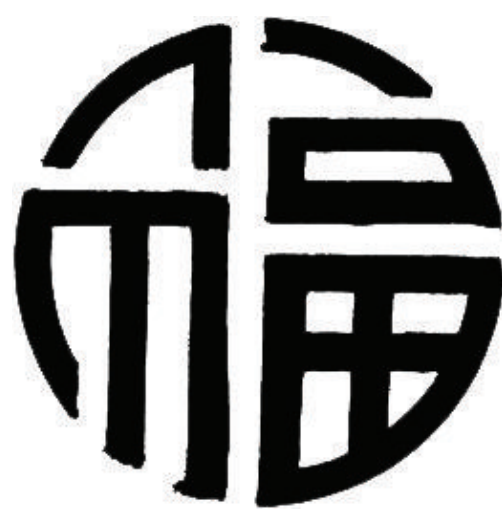

(a) Plain image

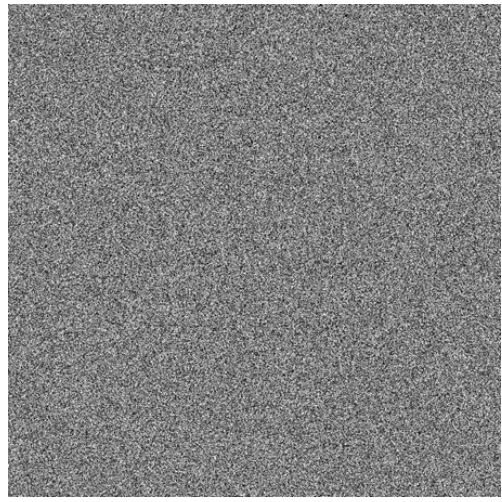

(c) Encrypted image

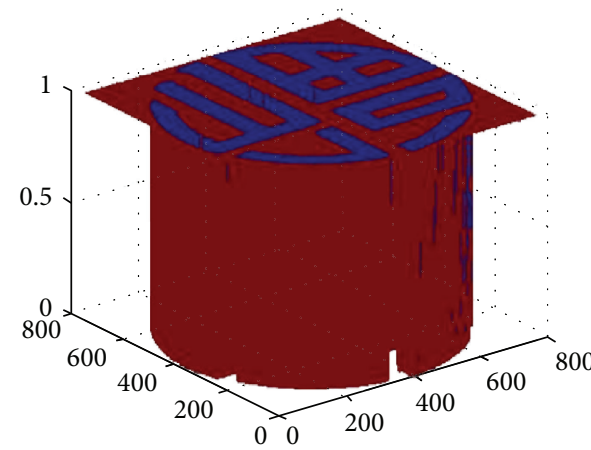

(b) Histogram of (a)

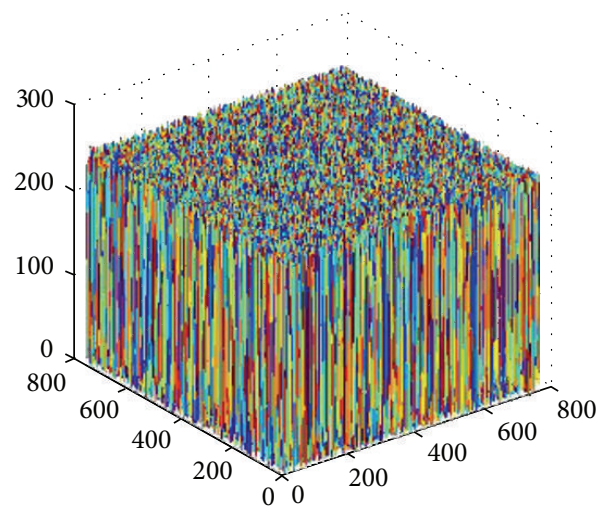

(d) Histogram of (c)

FIGURE 5: The binary image with size of $256 \times 256$.

TABLE 3: The entropy of plain image and its corresponding encrypted image.

\begin{tabular}{lccccccccc}
\hline & & \multicolumn{3}{c}{ Gray images } & \multicolumn{3}{c}{ Color baboon } \\
& Girl & Lena & Boat & Binary & Elaine & Eight fine horses & Red & Green & Blue \\
\hline Plain image & 7.7256 & 7.5769 & 7.1951 & 1.0060 & 7.5030 & 6.9958 & 7.7532 & 7.4642 & 7.7722 \\
Encrypted image & 7.9887 & 7.9898 & 7.9910 & 7.9916 & 7.9915 & 7.9905 & 7.9916 & 7.9917 & 7.9915 \\
\hline
\end{tabular}

their effects are represented in Figure 8. When the complete synchronization has not yet been achieved, the decryption effect is better with more abandoned points unsynchronized. Subtle difference between plain image and decrypted image cannot be observed subjectively. To avoid uncertainty of subjective evaluation, subjective evaluation is necessary for recovery performance. Common objective indexes are Mean Square Error (MSE) and Peak Signal to Noise Ratio (PSNR) defined as follows:

$$
\begin{aligned}
\text { MSE } & =\frac{1}{M \cdot N} \sum_{i=1}^{M} \sum_{j=1}^{N}\|P(i, j)-I(i, j)\|^{2}, \\
\text { PSNR } & =10 \cdot \log _{10}\left(\frac{\mathrm{MAX}_{I}^{2}}{\mathrm{MSE}}\right) .
\end{aligned}
$$

MSE is Mean Square Error between plain image $P$ and processed image $I$; $\mathrm{MAX}_{I}$ indicates that the maximum number of image colors is 255 with 8 bits per sample. PSNR is essentially identical to MSE, which is the logarithmic representation of MSE. However, MSE has poor correlation with subjective evaluation, so PSNR is usually used as evaluation index. PSNR is inversely proportional to effect of image encryption. When PSNR is more than $28 \mathrm{~dB}$, the decrypted image has better quality. In fact it cannot even distinguish difference with the naked eye when PSNR is up to [35 dB, $40 \mathrm{~dB}$ ]. After abandoning 0 points, 1000 points, 3000 points, 5000 points, and 7000 points, MSE and PSNR of decrypted gray are listed in Table 4. Comparing with the encrypted Lena, abandoning 7000 points has minimal distortion. 

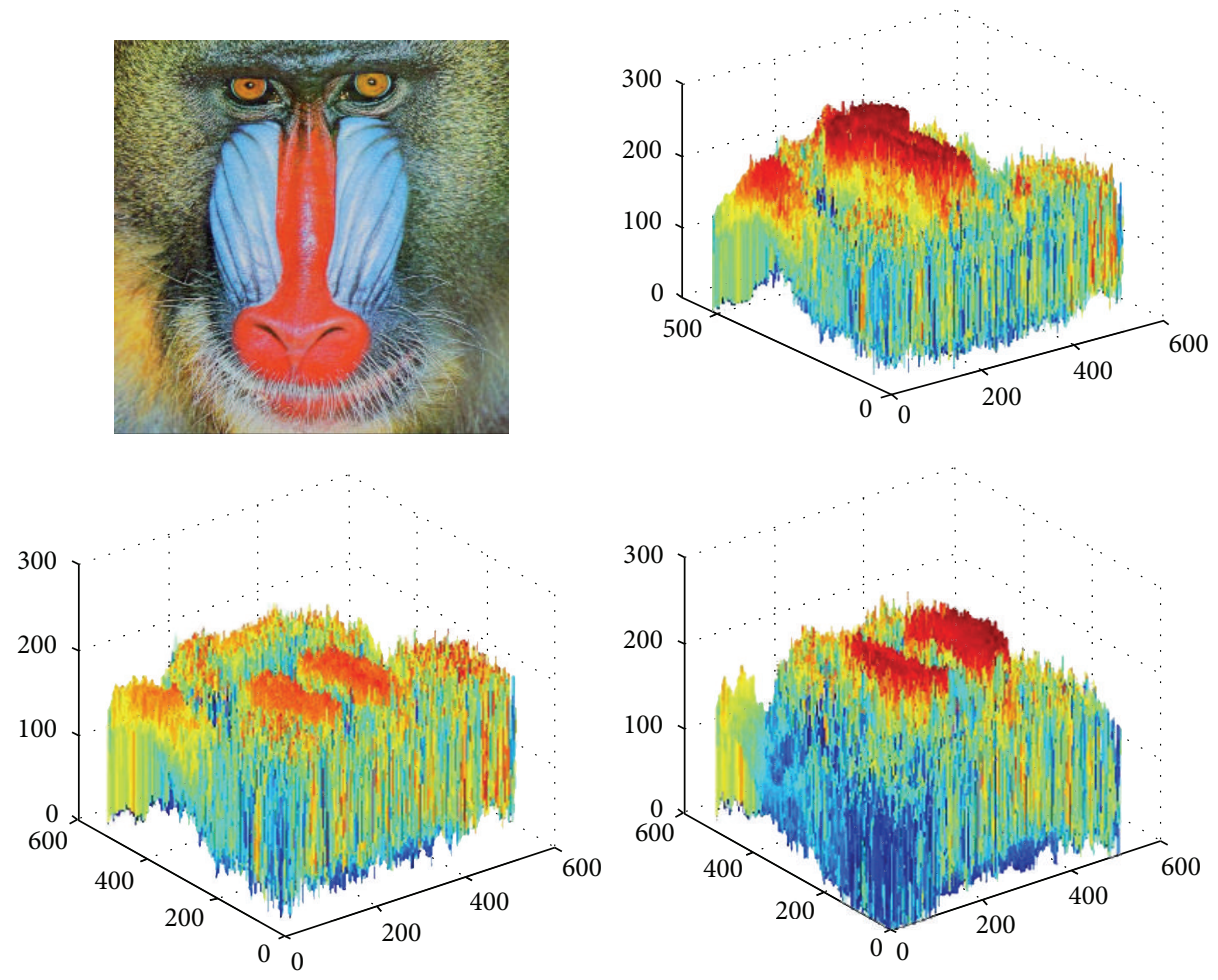

(a)
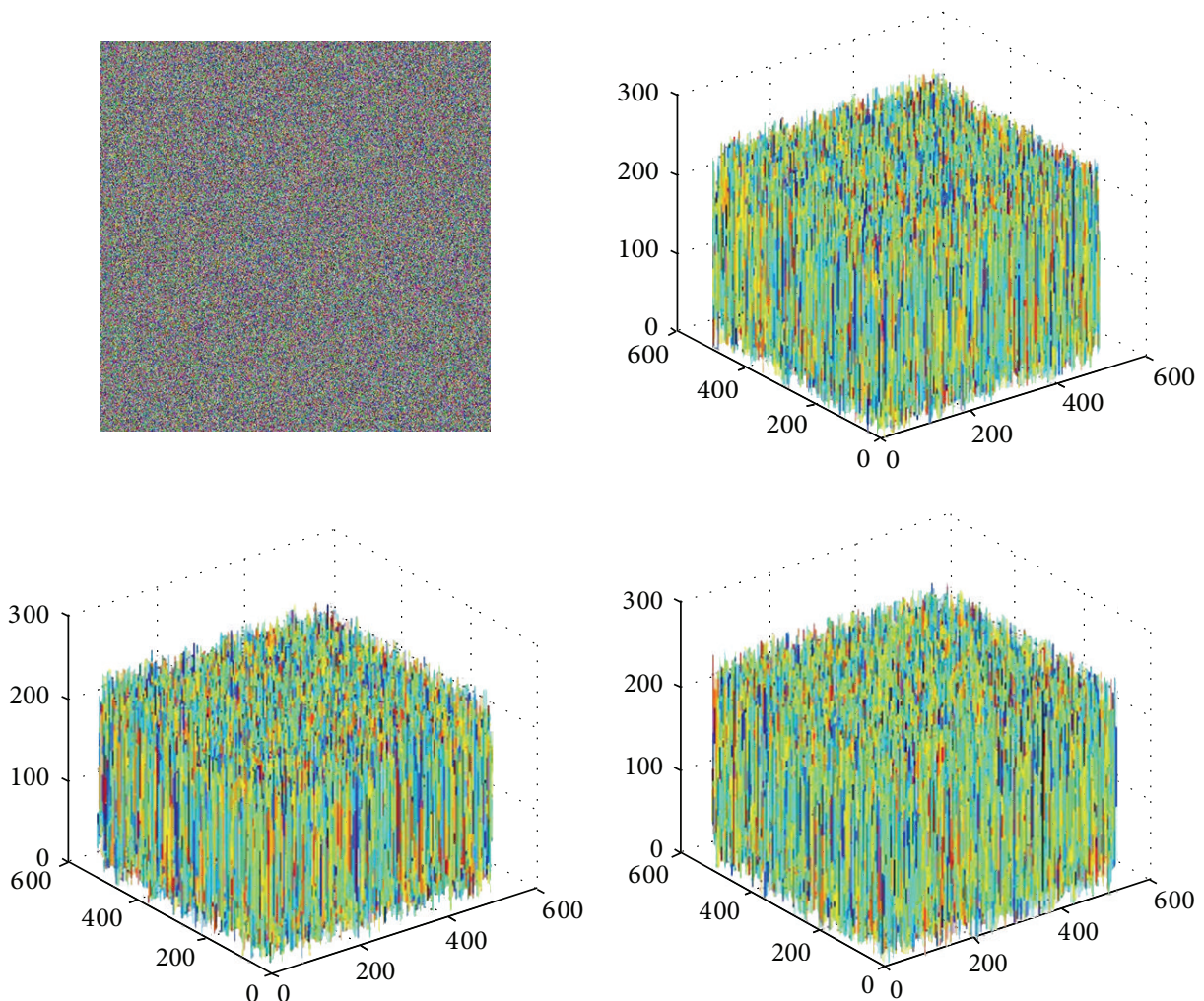

(b)

FIGURE 6: The color parrot image: (a) the plain image and histograms of its RGB segments and (b) the encrypted image and histograms of its RGB segments. 

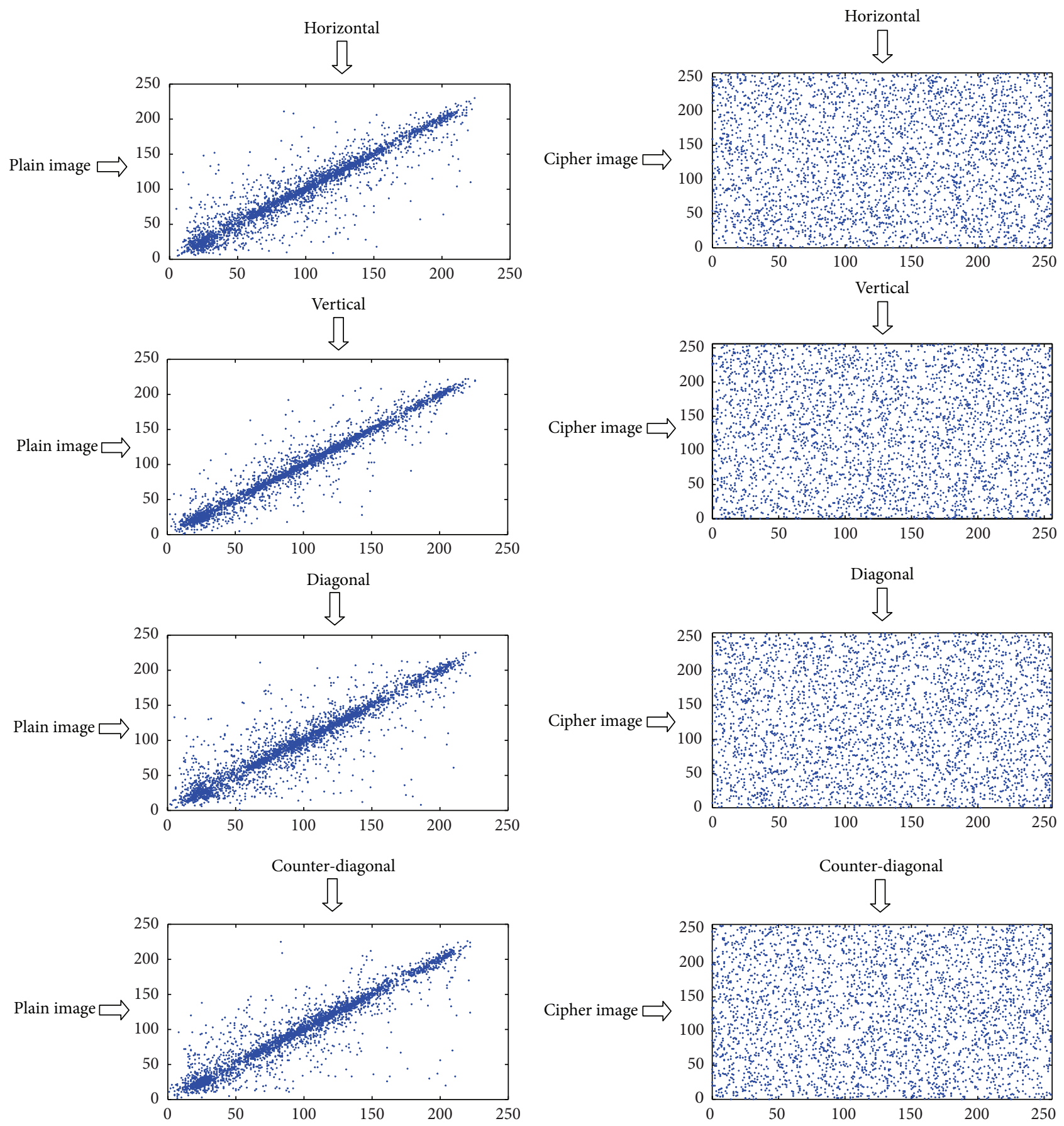

(a)

(b)

FIGURE 7: Correlation distribution of adjacent pixels: (a) plain Lena image and (b) encrypted Lena image.

4.4. Anti-Interference Attack. During transmission and other treatments, it is subjected to interferences and attacks. The anti-interference attack is an important indicator of testing the quality of the decryption algorithm. Encrypted images with $25 \%$ occlusion and $50 \%$ occlusion are represented in Figures 9(a) and 9(b); their decrypted images are shown in Figures 9(d) and 9(e), respectively. It is clear that the occlusion parts are diffused uniformly to the whole image; therefore algorithm of the paper has strong capability against cropping operation.

If the encrypted image is added, salt-and-pepper noise is seen with noise density 0.002 , when using the same key to restore the attacked image; the decrypted images are shown in Figure 9(f). It is clear that the encryption algorithm for saltand-pepper noise attack has good ability to resist interference attack. 


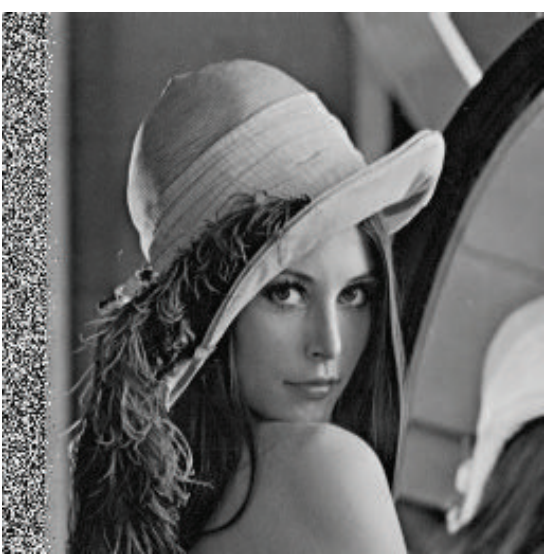

(a) Reject 0 points

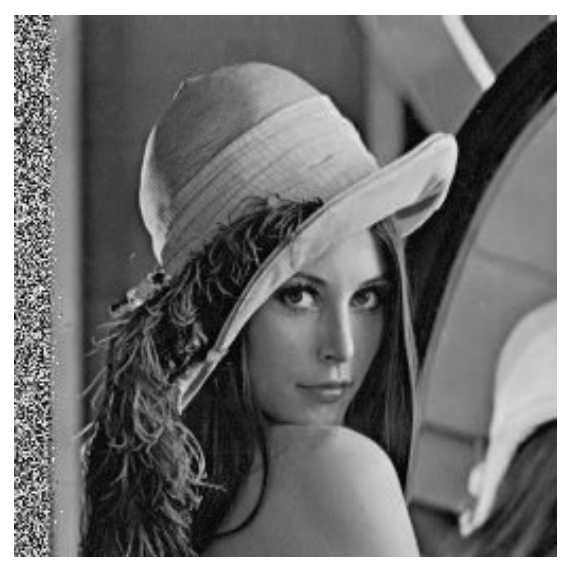

(b) Reject 1000 points

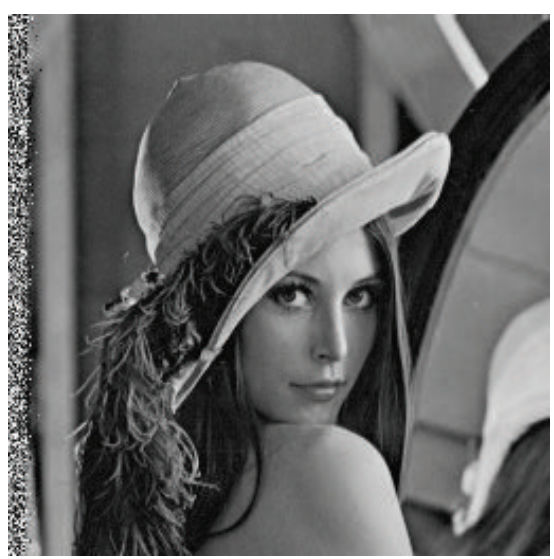

(c) Reject 3000 points

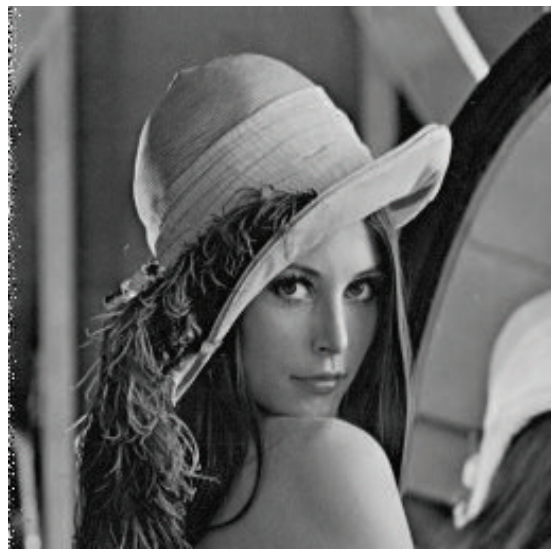

(d) Reject 5000 points

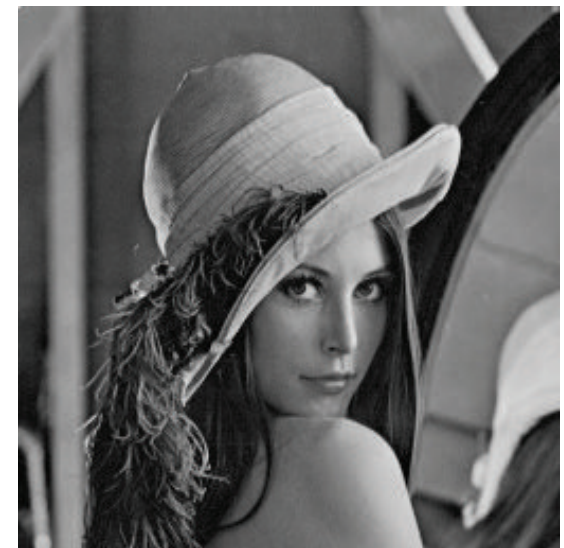

(e) Reject 7000 points

Figure 8: Decryption images of Lena with different dispose points.

TABLE 4: MSE and PSNR of different encrypted images under different reject points.

\begin{tabular}{lcccccc}
\hline Value & \multicolumn{3}{c}{ Reject points } & \multicolumn{2}{c}{$\begin{array}{c}\text { Encrypted } \\
\text { Lena }\end{array}$} \\
\hline MSE & 0 & 1000 & 3000 & 5000 & 9000 & 0.1208 \\
PSNR & 733.9676 & 648.8245 & 372.7224 & 62.9877 & 5009.3366 \\
\hline
\end{tabular}

\section{Conclusion}

Linear feedback controller is designed to synchronize the laser chaos and has higher precision. Steady-state error of synchronization reached $10^{-14}$. Based on synchronization a novel encryption algorithm is designed. Numerical simulation results show that the given algorithm has sufficiently large key space, highly sensitive keys, better pixel distribution characteristics, and good performance against ciphertextonly attack, differential attack, chosen plaintext attack, and statistical attack. The algorithm can be widely used in secure communication of multimedia data. Linear feedback controller has convenient operation and strong applicability and is suitable for industrial application. The synchronization of chaotic laser systems is applied in image encryption transmission and achieved good results. The algorithm can realize complete encryption in sender and lossless decryption in the receiver, so as to achieve digital image encryption transmission function.

\section{Competing Interests}

The authors declare that they have no competing interests.

\section{Acknowledgments}

This research is supported by the National Natural Science Foundation of China (no. 11501525) and the Aeronautical Science Foundation of China (no. 2013ZD55006). 


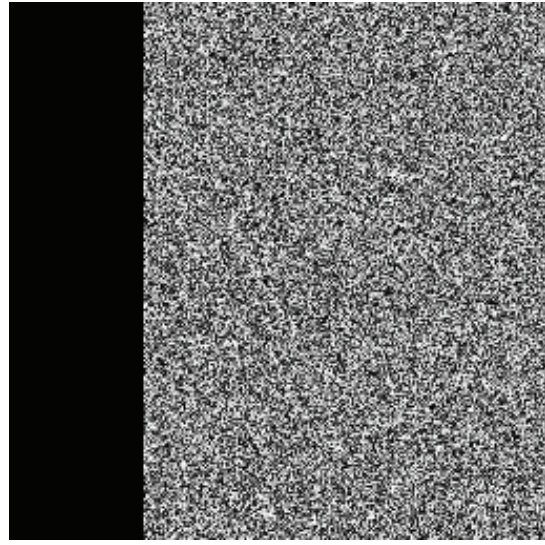

(a)

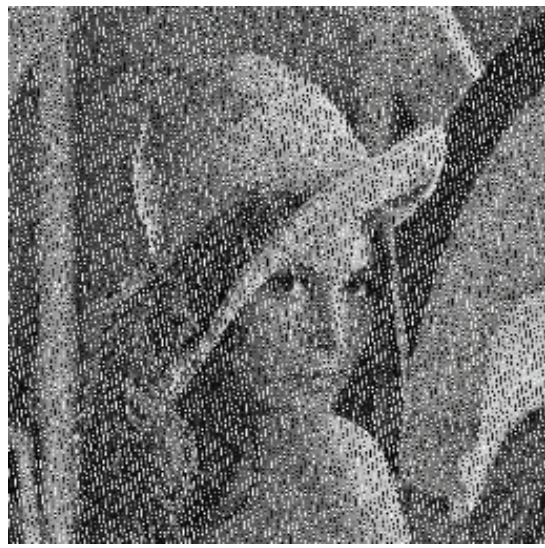

(d)

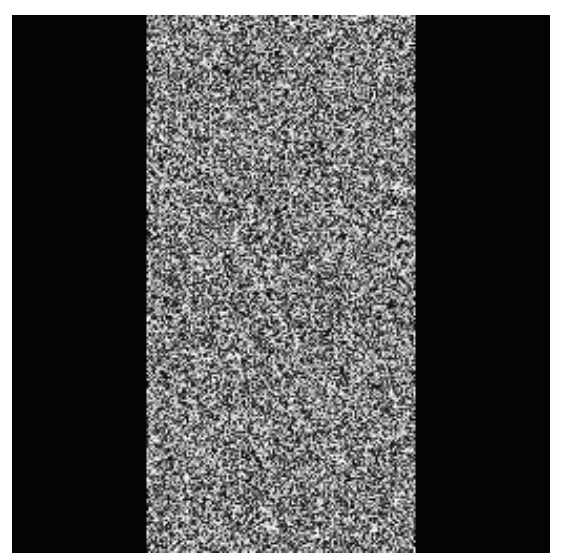

(b)

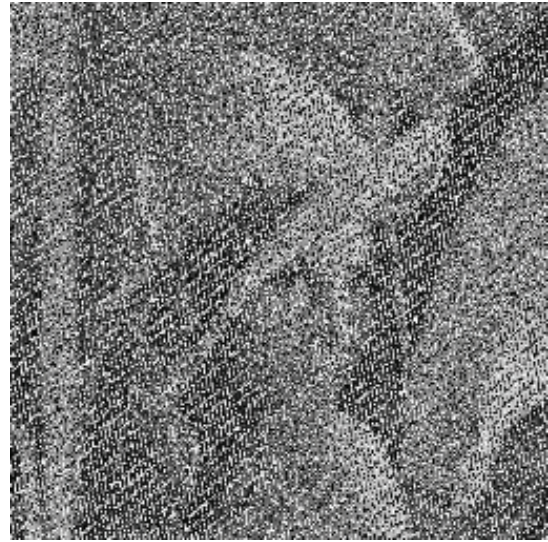

(e)

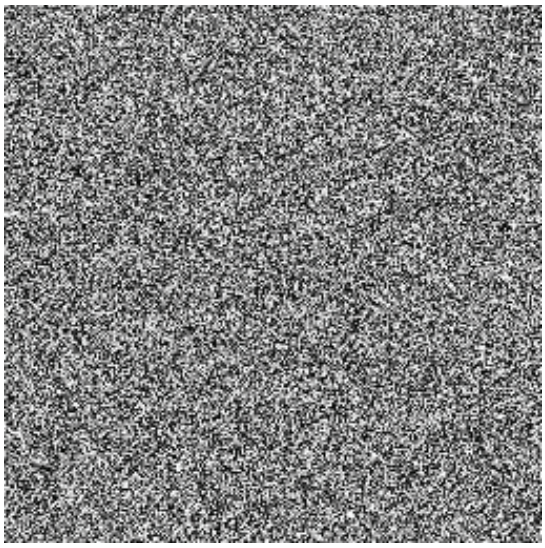

(c)

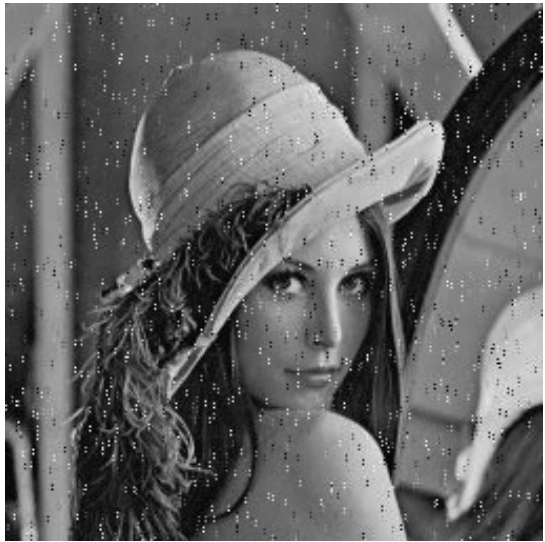

(f)

Figure 9: Encrypted image with (a) 25\% occlusion, (b) 50\% occlusion, and (c) salt-and-pepper noise; (d) decrypted image from (a), (e) decrypted image from (b), and (f) decrypted image from (c).

\section{References}

[1] R. Matthews, "On the derivation of a 'chaotic' encryption algorithm," Cryptologia, vol. 13, no. 1, pp. 29-42, 1989.

[2] G. R. Chen, Y. B. Mao, and C. K. Chui, "A symmetric image encryption scheme based on 3D chaotic cat maps," Chaos, Solitons and Fractals, vol. 21, no. 3, pp. 749-761, 2004.

[3] S. Behnia, A. Akhshani, H. Mahmodi, and A. Akhavan, "A novel algorithm for image encryption based on mixture of chaotic maps," Chaos, Solitons and Fractals, vol. 35, no. 2, pp. 408-419, 2008.

[4] C. X. Zhang, S. M. Yu, and G. R. Chen, "Design and implementation of compound chaotic attractors," International Journal of Bifurcation and Chaos, vol. 22, no. 5, Article ID 1250120, 13 pages, 2012.

[5] G. D. Ye and K.-W. Wong, "An efficient chaotic image encryption algorithm based on a generalized Arnold map," Nonlinear Dynamics, vol. 69, no. 4, pp. 2079-2087, 2012.

[6] X. J. Wu, C. X. Bai, and H. B. Kan, "A new color image cryptosystem via hyperchaos synchronization," Communications in Nonlinear Science and Numerical Simulation, vol. 19, no. 6, pp. 1884-1897, 2014.
[7] J. F. Zhao, S. Y. Wang, Y. X. Chang, and X. F. Li, "A novel image encryption scheme based on an improper fractionalorder chaotic system," Nonlinear Dynamics, vol. 80, no. 4, pp. 1721-1729, 2015.

[8] P. Colet and R. Roy, "Digital communication with synchronized chaotic lasers," Optics Letters, vol. 19, no. 24, pp. 2056-2058, 1994.

[9] L. Wu and S. Q. Zhu, "Multi-channel communication using chaotic synchronization of multi-mode lasers," Physics Letters A: General, Atomic and Solid State Physics, vol. 308, no. 2-3, pp. 157-161, 2003.

[10] J. M. Buldú, J. García-Ojalvo, and M. C. Torrent, "Multimode synchronization and communication using unidirectionally coupled semiconductor lasers," IEEE Journal of Quantum Electronics, vol. 40, no. 6, pp. 640-650, 2004.

[11] Y.-D. Chu, X.-F. Li, J.-G. Zhang, and Y.-X. Chang, "Nonlinear dynamics analysis of a modified optically injected semiconductor lasers model," Chaos, Solitons and Fractals, vol. 41, no. 1, pp. 14-27, 2009.

[12] L. Glass and M. C. Mackey, From Clocks to cHaos: The Rhythms of Life, Princeton University Press, Princeton, NJ, USA, 1988.

[13] A. T. Winfree, The Geometry of Biological Time, vol. 12, Springer, New York, NY, USA, 1990. 
[14] A. Y. Leung, X.-F. Li, Y.-D. Chu, and X.-B. Rao, "A simple adaptive-feedback scheme for identical synchronizing chaotic systems with uncertain parameters," Applied Mathematics and Computation, vol. 253, pp. 172-183, 2015.

[15] M. Mamat, W. S. Mada Sanjaya, and D. S. Maulana, "Numerical simulation chaotic synchronization of Chua circuit and its application for secure communication," Applied Mathematical Sciences, vol. 7, no. 1-4, pp. 1-10, 2013.

[16] S. Banerjee, L. Rondoni, S. Mukhopadhyay, and A. P. Misra, "Synchronization of spatiotemporal semiconductor lasers and its application in color image encryption," Optics Communications, vol. 284, no. 9, pp. 2278-2291, 2011.

[17] C. K. Volos, I. M. Kyprianidis, and I. N. Stouboulos, "Image encryption process based on chaotic synchronization phenomena," Signal Processing, vol. 93, no. 5, pp. 1328-1340, 2013.

[18] A. Kumar and M. K. Ghose, "Extended substitution-diffusion based image cipher using chaotic standard map," Communications in Nonlinear Science and Numerical Simulation, vol. 16, no. 1, pp. 372-382, 2011.

[19] C. K. Huang, C. W. Liao, S. L. Hsu, and Y. C. Jeng, "Implementation of gray image encryption with pixel shuffling and graylevel encryption by single chaotic system," Telecommunication Systems, vol. 52, no. 2, pp. 563-571, 2013.

[20] X. S. Luo, Theory and Methods of Chaos Control Synchronization and Its Applications, Guangxi Normal University Press, Guilin, China, 1st edition, 2007 (Chinese).

[21] C. E. Shannon, "Communication theory of secrecy systems," The Bell System Technical Journal, vol. 28, no. 4, pp. 656-715, 1949. 


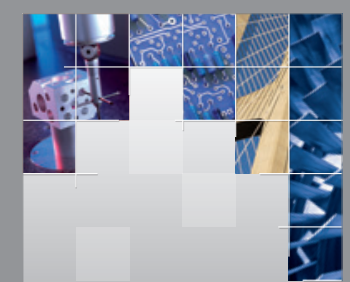

\section{Enfincering}
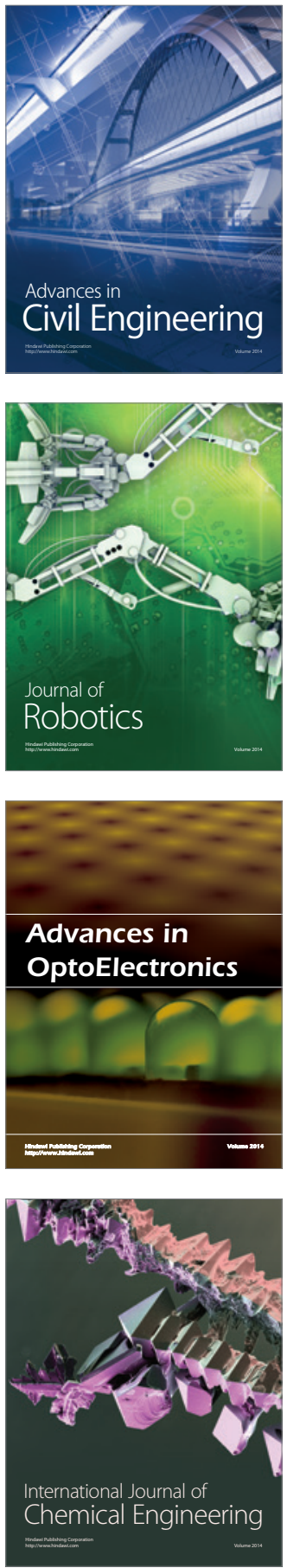

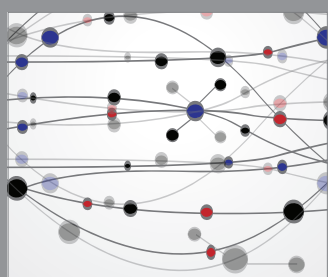

The Scientific World Journal

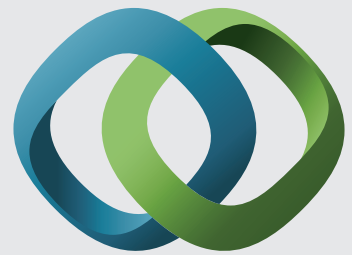

\section{Hindawi}

Submit your manuscripts at

http://www.hindawi.com
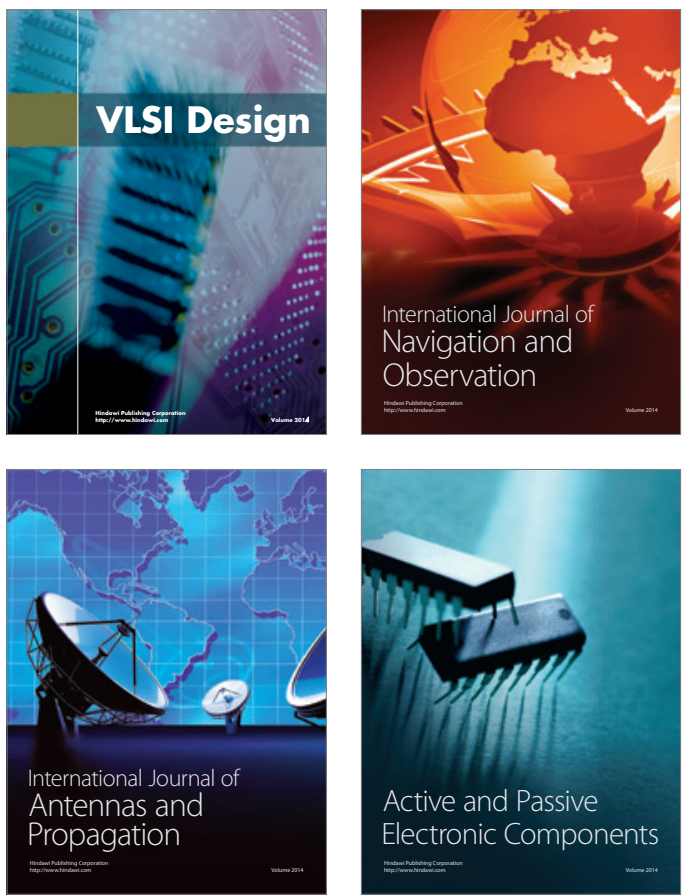
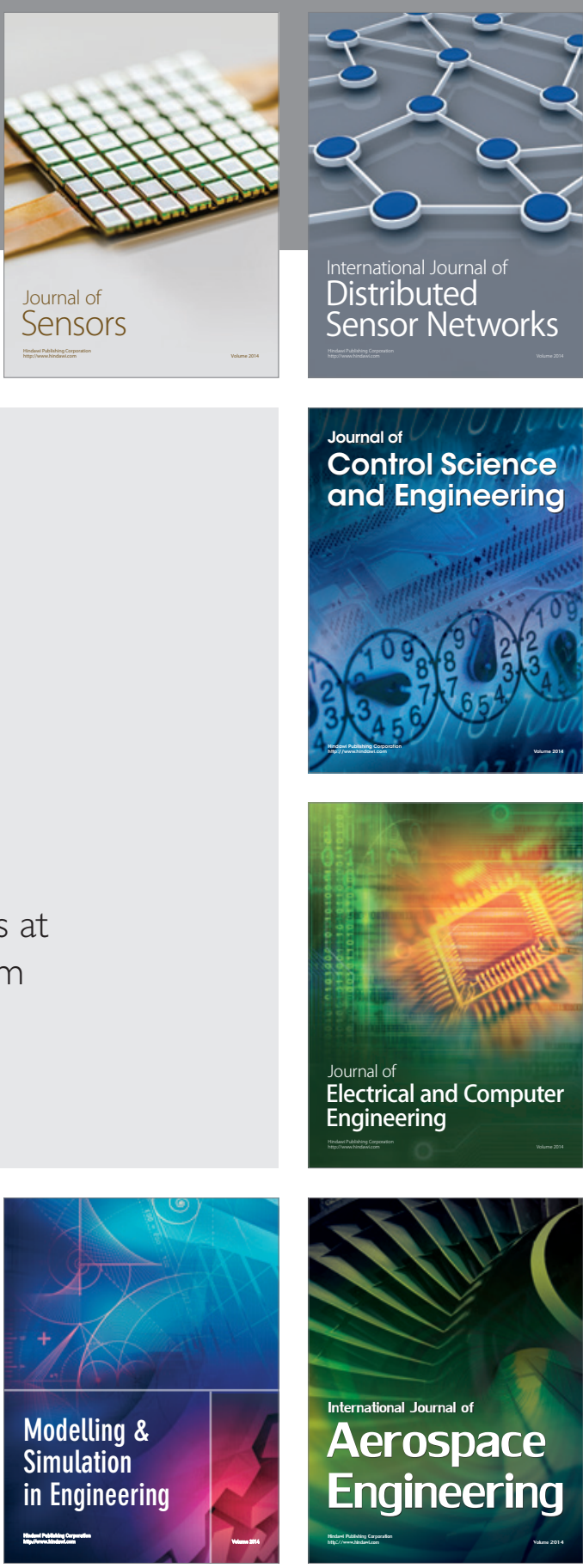

International Journal of

Distributed

Sensor Networks

Journal of

Control Science

and Engineering
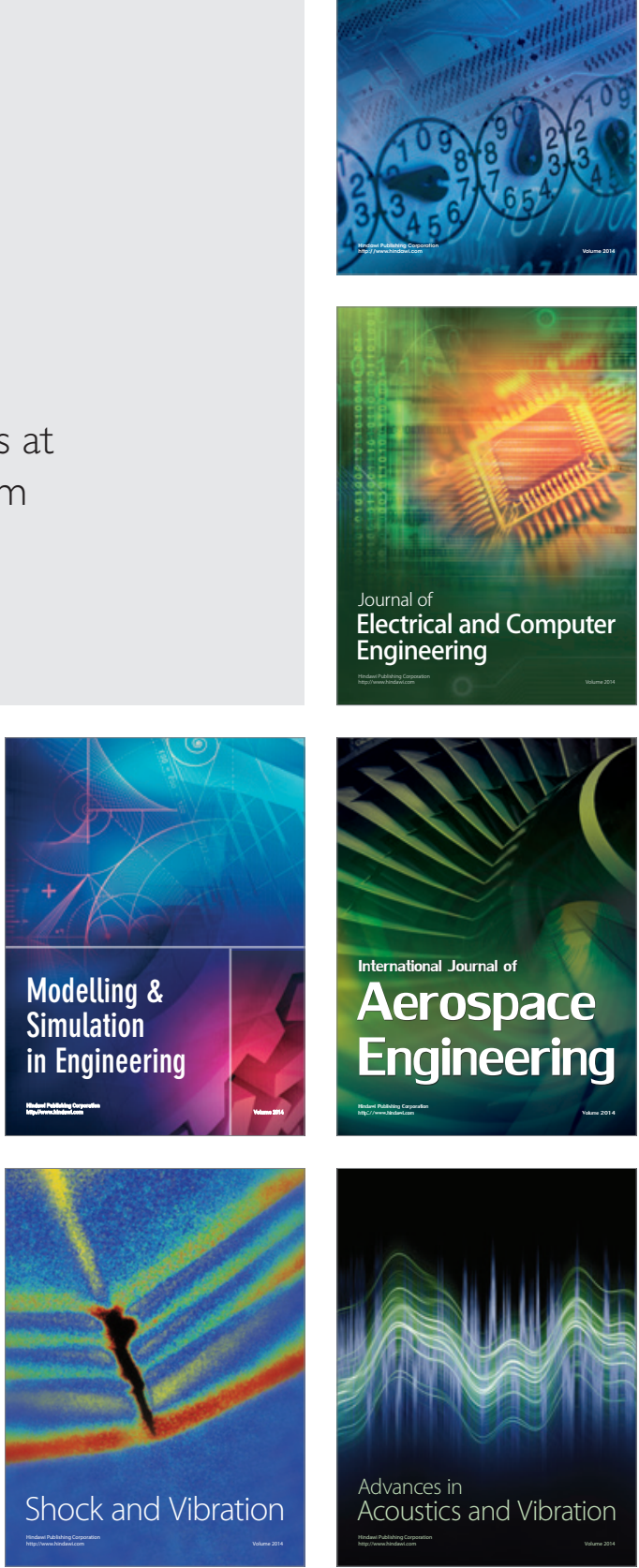